\title{
Out-of-Band Power Suppression for Cyclic SLM with Delayed Correlation and Matched Filter
}

\author{
Panca Dewi Pamungkasari ${ }^{* \dagger}$, Filbert H. Juwono ${ }^{\ddagger}$, Nur Hayati ${ }^{\dagger}$, and Mohammad Ghavami ${ }^{\S}$ \\ ${ }^{*}$ Graduate School of Environment and Information Sciences, Yokohama National University, Yokohama 240-8501, Japan \\ $\dagger$ Department of Communication Technology and Informatics, Universitas Nasional, Jakarta 12520, Indonesia \\ $\ddagger$ Department of Electrical and Computer Engineering, Curtin University Malaysia, 98009 Miri, Sarawak, Malaysia \\ $\S$ School of Engineering, London South Bank University, London SE1 0AA, United Kingdom
}

\begin{abstract}
Orthogonal frequency-division multiplexing (OFDM) has been used in many communication systems. However, OFDM suffers from undesirable high peak-to-average power ratio (PAPR) as it uses inverse discrete Fourier transform (IDFT) operation at the transmitter. One of the simplest schemes of the PAPR reduction is the cyclic-selective mapping (SLM). This paper presents cyclic-SLM without side information (SI) which employs delayed correlation with a matched filter (DC-MF) at the receiver. The cyclic-SLM combines the original OFDM signal and its cyclically shifted ones. In the previous conventional cyclic-SLM, the cyclically shifted signal samples are multiplied by a phase sequence which is drawn from Barker code sequence. However, this scheme results in higher out-of-band (OOB) power leakage. In this paper, we propose to use different phase sequence for each OFDM symbol in one OFDM frame to overcome the OOB problem. As a consequence, multiple matched filters are used at the receiver. Simulation results show that the proposed method has the same OOB as the conventional OFDM. In addition, we also analyze the performance of the proposed scheme in terms of the PAPR reduction and bit error rate (BER).
\end{abstract}

Index Terms-SLM, PAPR, delayed correlation, matched filter, OOB power.

\section{INTRODUCTION}

Many modern wired and wireless broadband communication system standards adopt orthogonal frequency-division multiplexing (OFDM) in the physical layer. We also note that OFDM and OFDM-based waveforms are among the candidates for 5G communication systems [1], [2]. OFDM is preferred as it offers several advantages such as high speed data transmission, high mobility, and high spectrum efficiency. Despite these advantages, OFDM system has a major issue of high peak-to-average power ratio (PAPR) which can degrade the system performance. A power amplifier (PA) is commonly used at the transmitter of a communication system. The nonlinear characteristic of the PA deliver the high signal samples to the saturation region and causes nonlinear distortions and spectrum spreading [3]. In order to reduce the PAPR of the OFDM system, many PAPR reduction schemes have been proposed and analyzed in [3], [4] and the references therein.

Selective mapping (SLM) technique, which is a distortionless PAPR reduction methods, has been popularly used in OFDM systems. However, SLM needs multiple inverse fast Fourier transform (IFFT) blocks at the transmitter which causes high computational complexity. To overcome this issue, several low-complexity SLM (LC-SLM) has been proposed in the literature. LC-SLM techniques with two IFFT blocks were proposed in [5]-[11]. Moreover, to further reduce the computational complexity, a cyclic-SLM scheme with only one IFFT has been proposed in [7]. The cyclic-SLM the original signal is added to its cyclically shifted and phase rotated versions.

The above-mentioned SLM techniques need to send side information (SI) to recover the original symbol sequence at the receiver side. In the cyclic-SLM, for example, SI contains information about cyclic shifts and rotation phases. Note that throughput degradation of the OFDM systems may occur due to the errors during the SI transmission to the receiver side. Protecting the SI transmission is one of the challenges in the SLM. On the other hand, several schemes have been proposed to avoid sending SI explicitly but embed it in the transmitted signal instead. Using this scheme, the receiver needs to recover the embedded SI with minimal error. Otherwise, it will cause distortion on the signal. Therefore, accuracy rate of the SI detection is needed as it is related to bit error rate (BER) performance [12]-[14].

Several schemes have been proposed to reduce PAPR without sending SI explicitly. In [12], the difference between the average energies of extended and non-extended symbols was used to recover the SI index at the receiver side, which made higher order modulation symbols influence the accuracy of the SI detection at the receiver. In [15], a scheme based on [12] was proposed. The possible phase vector was prepared at the receiver side and an SI index was embedded in transmitted signal which decreased the throughput of the OFDM systems. In [13], the cyclic-SLM with delayed correlation (DC) was proposed to reduce the PAPR and estimate the cyclic shift at the receiver side. The proposed solution had trade off between PAPR reduction and bit error rate (BER). In [14], a method to improve the performance of the previous scheme [13] was proposed. It implemented cyclic-SLM with 3 branches and appended a matched filter (MF) with Barker code sequences to estimate the cyclic shift at the receiver side. In [16], the effect of cyclic shift resolution was investigated. The proposed cyclicSLM in [13], [14], [16], however, increased the out-of-band (OOB) power leakage. This paper aims to suppress the OOB power leakage of the cyclic-SLM scheme by using different phase sequence for each OFDM symbol in a frame. We will also analyze the performance.

The time domain cyclic-SLM scheme with delayed correla- 
tion and matched filtering (DC-MF) is discussed in this paper. It employs only one IFFT, avoids to send the SI explicitly, and uses different phase sequences at the transmitter and hence multiple matched filters at the receiver to overcome the OOB problem. The lowest PAPR of the signal candidates (SCs) which are generated by specific phase sequence is chosen. The data symbols at the receiver side is recovered by using DC-MF operation. The receiver can estimate the amount of the cyclic shift by examining the interval between the peaks at the outputs of the DC-MF operation.

The rest of paper is organized as follows. Section II discusses system model, OFDM symbol structure, the cyclic-SLM in the time domain, OOB power, the proposed of cyclic shift estimation scheme, channel estimation, and minimum mean square error (MMSE) detection. Section III presents the simulation results of the proposed scheme and finally Section IV draws some conclusions.

\section{SySTEM MODEL}

The OFDM signal samples, $x[n]$, at time index $n$ can be expressed by

$$
x[n]=\frac{1}{N} \sum_{k=0}^{N-1} X[k] \exp \left(j \frac{2 \pi n k}{N}\right), 0 \leq n \leq N-1,
$$

where $X[k]$ is the data symbol at the $k^{\text {th }}$ subcarrier, $k$ is the subcarrier index, and $N$ is the number of the subcarriers.

Before being transmitted, a guard interval (GI) in terms of a cyclic prefix is prepended to the OFDM symbol. The GI is a copy of a fraction of the last part of the OFDM signal. Mathematically, the OFDM signal with a GI is expressed by

$x_{f}[n]= \begin{cases}x[N+n], & n=-N_{G I},-N_{G I}+1, \ldots,-1 \\ x[n], & n=0,1, \ldots, N-1,\end{cases}$

where $N_{G I}$ is the length of the GI.

\section{A. Cyclic-SLM in Time Domain}

Cyclic-SLM [7] has been proposed to reduce the complexity of the conventional SLM by introducing the cyclic shift and phase shift to generate the SCs. As a result, it requires only one IFFT and reduces the complexity while maintaining the same performance. Mathematical expression of the cyclic-SLM SCs in the time domain is given by

$$
x_{d}\left[n, \Delta_{d}\right]=\left\{\begin{array}{l}
x\left[N-\Delta_{d}+n\right], \quad 0 \leq n \leq \Delta_{d}-1, \\
x\left[n-\Delta_{d}\right], \quad \Delta_{d} \leq n \leq N-1,
\end{array}\right.
$$

where $\Delta_{d}$ is the amount of the cyclic shift for the $d^{\text {th }} \mathrm{SC}$ and $\Delta_{d} \in\{C \delta\}$ where $C$ is an integer. The receiver needs to estimate the amount of the cyclic shift in order to reconstruct the transmitted signal. The cyclic shift resolution, $\delta$, plays an important role in determining the accuracy of the cyclic shift estimation. Large enough resolution is needed to obtain an acceptable performance.
The transmitted signal, $s[n]$, is the superposition of the SCs and the original signal which can be expressed by

$$
s[n]=x_{f}[n]+\sum_{d=1}^{D} Q_{d} x_{f}\left[n, \Delta_{d}\right]
$$

where $D$ is the number of branches (SCs), $Q_{d}$ is the $d^{\text {th }}$ element of the phase sequence, and $x_{f}[n, \Delta]$ is the SC sequences with the GI given by

$$
x_{f}\left[n, \Delta_{d}\right]= \begin{cases}x_{d}\left[n, \Delta_{d}\right], & 0 \leq n \leq N-1, \\ x_{d}\left[N+n, \Delta_{d}\right], & -N_{G I} \leq n<0,\end{cases}
$$

for $0<\Delta_{d}<N-N_{G I}$. Note that the previous research papers [13], [14], [16] use Barker code sequence with length $3,[1,1,-1]$, as the phase sequence for each OFDM symbol. In this paper, we use different phase sequence for each OFDM symbol in a frame to suppress the OOB power level.

The resulting $s[n]$ in (4) with the lowest PAPR is selected and transmitted. It is worth mentioning that the PAPR is calculated over $L$ consecutive OFDM symbols. As each branch has different amount of the cyclic shifts $\Delta_{d}$, we need to calculate the PAPR for every $\Delta_{d}$ value to select the lowest average PAPR.

\section{B. OOB Power Spectral Density}

PAPR drives the signal to the non-linear region of the PA, which causes undesirable increase of the side lobes level of the power spectral density (PSD), in-band noise, and OOB radiation [3]. It involves spectral spreading which will be the interference between adjacent channels. PSD calculation is conducted by using the following equations

$$
\begin{gathered}
s_{a}[k]=\sum_{n=0}^{N+N_{G I}} s[n] \exp \left(j \frac{2 \pi n k}{N+N_{G I}}\right), \\
P_{\mathrm{avg}}=\frac{1}{M} \sum_{m=1}^{M}\left|s_{a}[k]\right| \\
P_{\max }=\max \left(\mathrm{P}_{\mathrm{avg}}\right), \\
P_{\mathrm{PSD}}(d B)=10 \log _{10} \frac{\mathrm{P}_{\mathrm{avg}}}{\mathrm{P}_{\max }}
\end{gathered}
$$

where $s_{a}[k]$ is the transmitted signal with the lowest PAPR in frequency domain, $M$ is the number of OFDM symbols, $P_{\text {avg }}$ is the average power over $M$ OFDM symbols, $P_{\max }$ is the maximum power, and $P_{\mathrm{PSD}} \mathrm{dB}$ is the PSD in $\mathrm{dB}$.

\section{Proposed Cyclic Shift Estimation Scheme}

As already mentioned, no SI is transmitted to recover the transmitted signal. Instead, the amount of the cyclic shift is estimated by using the DC-MF at the receiver side. At the receiver, the received signal samples can be expressed by

$$
r[n]=\sum_{i} h[i] s[n-i]+w[n]
$$


where $h[i]$ represents the impulse response of the time-varying multipath channel and $w[n]$ is additive white Gaussian noise (AWGN) with zero mean and variance of $\sigma^{2}$ and is independent of the input symbols. In this paper, channel estimation using preamble symbols is performed and will be discussed in following subsection.

The output of the DC operation is the product of the received signal (in time domain) and its conjugate of the last part of the received OFDM symbol. The DC operation is described as follows

$$
\begin{aligned}
c[n]= & r[n] r^{*}[n-N] \\
= & \left(\sum_{i} h[i] s[n-i]+w[n]\right) \times \\
& \left(\sum_{i^{\prime}} h^{*}\left[i^{\prime}\right] s^{*}\left[n-N-i^{\prime}\right]+w^{*}[n-N]\right),
\end{aligned}
$$

where $r[n]$ denotes the received symbol in the time domain, $r^{*}[n-N]$ is the conjugate of the GI, $s[n-i]=s^{*}\left[n-N-i^{\prime}\right]$, and $c[n]$ is given by (12) on the next page.

The outputs of the multiplier are summed over the period of the GI, $N_{\text {GI }}$. After the summation, the outputs of the DC is averaged over the corresponding samples of OFDM symbol as follows

$$
C[n]=\frac{1}{L} \sum_{l=1}^{L} \sum_{p=0}^{N_{G I}-1} c\left[n-l\left(N+N_{G I}\right)-p\right],
$$

where $p$ is the index for integration. When the GI is multiplied by the its conjugate which was previously added as GI, the highest peak occurs. The first peak corresponds to the GI of the OFDM symbols and is calculated by using the following maximization problem

$$
n^{\max }=\underset{n}{\arg \max }|C[n]| .
$$

As the GI comprises the last part of the original OFDM symbol sequence, $\left[x\left[N-N_{G I}\right] \ldots x[N]\right]$ and the last part of the SC sequence, $\left[x\left[N-N_{G I}-\Delta_{d}\right] \ldots x\left[N-1-\Delta_{d}\right]\right]$, we can use DC output to examine the correlation between the GI sequence and the received signal with the delay of $\hat{\Delta}_{d}$ which is denoted by $r\left[n-\hat{\Delta}_{d}\right]$. Furthermore, if $\hat{\Delta}_{d}=\Delta_{d}$, the delayed correlation also produces another peak as explained in the following equation

$$
\hat{c}_{d}\left[n^{\max }-\hat{\Delta}_{d}\right]=r\left[n^{\max }-\hat{\Delta}_{d}\right] r^{*}\left[n^{\max }-N\right],
$$

where $\hat{\Delta}_{d}$ is the candidate for the amount of the cyclic shift on the $d^{\text {th }}$ branch. We see that (15) can be rewritten as

$$
\begin{aligned}
\hat{c}_{d}\left[n^{\max }-\hat{\Delta}_{d}\right] \\
=\left(\sum_{i} h[i] s\left[n^{\max }-\hat{\Delta}_{d}-i\right]+w\left[n^{\max }-\hat{\Delta}_{d}\right]\right) \\
\quad \times\left(\sum_{i^{\prime}} h^{*}\left[i^{\prime}\right] s^{*}\left[n^{\max }-N-i^{\prime}\right]+w^{*}\left[n^{\max }-N\right]\right) .
\end{aligned}
$$

Thus, after the integration, the DC outputs the following

$$
\hat{C}_{d}\left[n^{\max }, \hat{\Delta}_{d}\right]=\frac{1}{L} \sum_{l=1}^{L} \sum_{p=0}^{N_{G I}-1} \hat{c}_{d}\left[n^{\max }-l\left(N+N_{G I}\right)-\hat{\Delta}_{d}-p\right] .
$$

The output of the DC is then passed to a matched filter to estimate the amount of the cyclic shift. To reduce the complexity, the matched filter is restricted to be every $\delta$ sample as $\{\Delta, \Delta+\delta, \ldots, \Delta+(d-1) \delta\}$. The structure of the matched filter with $(\delta-1)$ delay line is depicted in Fig. 1. Note that as we have different phase sequence for each OFDM symbol, correspondingly we have multiple numbers of matched filter.

The output of the MF is given by

$$
\hat{C}_{m}\left[n^{\max }, \hat{\Delta}\right]=\sum_{d=1}^{D} Q_{d} \hat{C}_{d}\left[n^{\max }, \hat{\Delta}+(\delta-1)(d-1)\right] .
$$

The following maximization problem is used to estimate the cyclic shift of the first branch, $\Delta^{\max }$.

$$
\Delta^{\max }=\underset{\hat{\Delta}}{\arg \max }\left|\hat{C}_{m}\left[n^{\max }, \hat{\Delta}\right]\right|
$$

After getting the cyclic shift of the first branch, the cyclic shift of the other branches can be easily calculated.

\section{Channel Estimation and Minimum Mean Square Error De- tection}

Preamble symbols can be used to estimate the channel requency response (CFR) and the coarse symbol timing. Commonly, preamble symbols, $s_{p}[n]$ are transmitted before the data symbols. The received preamble symbols, $r_{p}[n]$ are given by

$$
r_{p}[n]=\sum_{i=0}^{N-1} h[i] s_{p}[n-i]+w[n]
$$

The received preamble symbols at the $k^{\text {th }}$ subcarrier, $R_{p}[k]$ are obtained by taking the $N$-point normalized FFT as

$$
R_{p}[k]=\sum_{n=0}^{N-1} r_{p}[n] e^{-j 2 \pi n k / N} .
$$

The estimated CFR at the $k^{\text {th }}$ subcarrier, $\hat{H}[k]$, is given by

$$
\hat{H}[k]=R_{p}[k] / S_{p}[k],
$$

where $S_{p}[k]$ is the transmitted preamble symbols in the frequency domain.

As the cyclic-SLM is performed in the time domain, the CFR needs to be converted to channel impulse response (CIR) as follows

$$
\hat{\mathbf{h}}=\operatorname{IFFT}\left(\hat{\mathbf{H}}^{\mathrm{T}}\right)=\left[\begin{array}{lll}
\hat{h}[0] & \ldots \hat{h}[N-1]
\end{array}\right]^{T},
$$

where $\hat{h}[i]$ is the CIR at the $i^{\text {th }}$ sample, $\hat{\mathbf{H}}=$

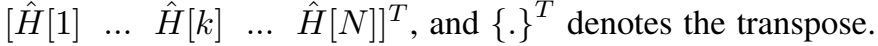
The CIR should be modified such that it takes into account the effect of the cyclic shift as follows

$$
\hat{h}_{d}[i]=\sum_{d=1}^{D}(\hat{h}[i]+\hat{h}[i-(\Delta+(\delta-1)(d-1)])
$$




$$
\begin{aligned}
c[n] & =\sum_{i}|h[i]|^{2}|s[n-i]|^{2}+\sum_{i} \sum_{i^{\prime}, i^{\prime} \neq i} h[i] s[n-i] h^{*}\left[i^{\prime}\right] s^{*}\left[n-N-i^{\prime}\right] \\
& +\sum_{i}(h[i] s[n-i]) w^{*}[n-N]+\sum_{i^{\prime}}\left(h^{*}\left[i^{\prime}\right] s^{*}\left[n-N-i^{\prime}\right] w[n]\right)+w[n] w^{*}[n-N] .
\end{aligned}
$$

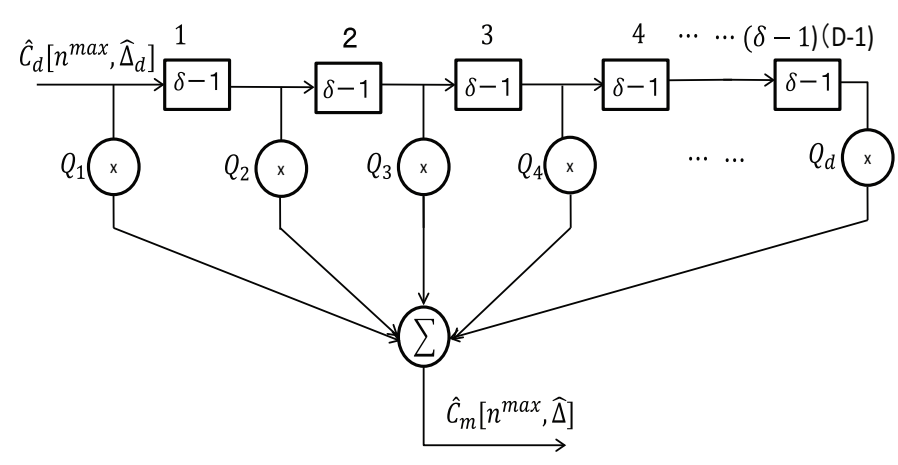

Fig. 1. Matched filter.

For the channel equalization, we use the minimum mean square error (MMSE) technique as it is robust to noise. The weight coefficient of the MMSE detection at the $k^{\text {th }}$ subcarrier, $W_{d}^{H}[k]$, is given by

$$
W_{d}^{H}[k]=\hat{H}_{d}^{H}[k]\left(\hat{H}_{d}[k] \hat{H}_{d}^{H}[k]+\sigma^{2}\right)^{-1},
$$

where $\hat{H}_{d}[k]$ is the CFR of $\hat{h}_{d}[i]$ given by

$$
\hat{H}_{d}[k]=\sum_{i=0}^{N-1} \hat{h}_{d}[i] e^{-j 2 \pi i k / N} .
$$

The demodulated signal at the $k^{\text {th }}$ subcarrier can be calculated by

$$
\hat{X}[k]=W_{d}^{H}[k] R[k]
$$

where $R[k]$ is the received signal in the frequency domain.

\section{Simulation Results}

The simulation conditions are given in Table I. It can be seen in the table that we use eight sets of phase sequences or MF coefficients for every 8 OFDM symbols per frame: [ $\left.\begin{array}{lll}1 & 1 & 1\end{array}\right]$, [1 $\left.\begin{array}{ll}1 & -1\end{array}\right],\left[\begin{array}{lll}1 & -1 & 1\end{array}\right],\left[\begin{array}{lll}1 & -1 & -1\end{array}\right],\left[\begin{array}{lll}-1 & 1 & 1\end{array}\right],\left[\begin{array}{lll}-1 & 1 & -1\end{array}\right],\left[\begin{array}{lll}-1 & -1 & 1\end{array}\right]$, and [-1 -1 $-1]$.

\section{A. PAPR Reduction}

The PAPR performance curves of the original OFDM signal and the cyclic-SLM scheme are obtained through computer simulation. They are evaluated by using complementary cumulative distribution functions (CCDFs). The number of the branches $D$, is set to be 3 , the resolution of the cyclic shift is $\delta=4$ or $\delta=8$, and the number of the symbols for averaging,

\begin{tabular}{|c|c|}
\hline Modulation & QPSK/OFDM \\
\hline FFT Size & 256 \\
\hline Number of Data Subcarriers & 128 \\
\hline Guard Interval & $0.25 \times$ FFT Size \\
\hline Number of Symbols for Averaging & $1,2,4,8$ Symbols \\
\hline Cyclic Shift $\left(\Delta_{d}\right)$ & $60-124$ \\
\hline Resolution of Cyclic Shift $(\delta)$ & 4 or 8 \\
\hline Coding Scheme & Convolutional Coding \\
\hline Interleaver & Matrix: $16 \times 8$ \\
\hline Decoding Scheme & Soft Decision Viterbi \\
\hline Constraint Length & 7 \\
\hline Coding Rate & $1 / 2$ \\
\hline Polynomial Generator & {$\left[\begin{array}{ll}171 & 133\end{array}\right]$} \\
\hline Number of Branches & 3 \\
\hline Channel & Uniform (12 paths) \\
\hline Phase sequences (Matched Filter) & $\begin{array}{l}{\left[\begin{array}{lll}1 & 1 & 1\end{array}\right],\left[\begin{array}{lll}1 & 1 & -1\end{array}\right],} \\
{\left[\begin{array}{lll}1 & -1 & 1\end{array}\right],\left[\begin{array}{lll}1 & -1 & -1\end{array}\right],} \\
{\left[\begin{array}{lll}-1 & 1 & 1\end{array}\right],\left[\begin{array}{lll}-1 & 1 & -1\end{array}\right],} \\
{\left[\begin{array}{lll}-1 & -1 & 1\end{array}\right],\left[\begin{array}{lll}-1 & -1 & -1\end{array}\right]}\end{array}$ \\
\hline
\end{tabular}
$L$ is $1,2,4$, and 8 . Figure 2(a) shows that the cyclic-SLM with $1,2,4$, and 8 symbols can reduce the PAPR by around $2.8 \mathrm{~dB}$,
TABLE I SimUlation PARAMETERS.

$2.7 \mathrm{~dB}, 2.6 \mathrm{~dB}$ and $2.5 \mathrm{~dB}$, respectively, as compared to that of the original signal at the CCDF of $10^{-4}$. Whereas in Fig. 2(b) the proposed scheme with 1, 2, 4, and 8 symbols achieves PAPR reduction of from 1.7 to $1.9 \mathrm{~dB}$ as compared to that of the original signal at the CCDF of $10^{-4}$. Figures 2(a) and 2(b) prove that the highest PAPR reduction takes place when the number of the symbols for averaging is 1 and the smallest PAPR reduction occurs when the number of the symbols for averaging is 8 . PAPR reduction with $\delta=4$ is around $0.8 \mathrm{~dB}$ better than that with $\delta=8$. It happens because the number of the sets of the cyclic shifts which indicates the number of SCs is larger with $\delta=4$ when the maximum cyclic shift is limited to 124 . Therefore, the opportunity to achieve lower PAPR is larger. In this scheme, the number of symbols for averaging and the resolution of cyclic shift, $\delta$, influences the amount of PAPR reduction though they do not bring over the computational complexity.

\section{B. OOB Performance}

Spectrum properties at the transmission power amplifier output after peak power reduction are important to evaluate 


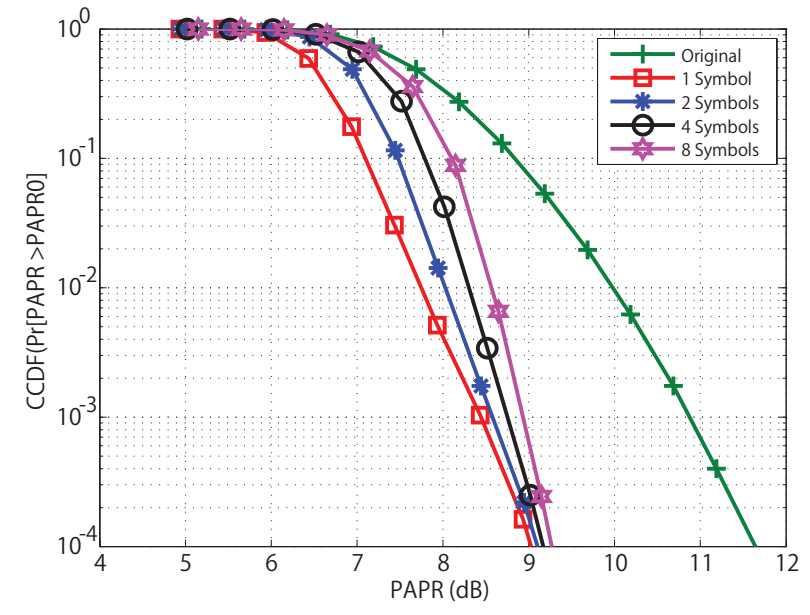

(a) Resolution of cyclic shift $\delta=4$

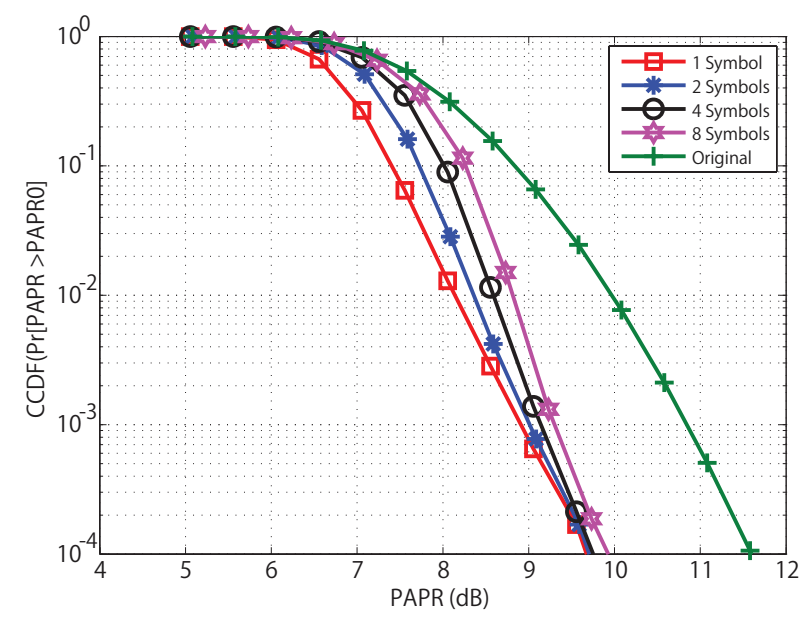

(b) Resolution of cyclic shift $\delta=8$

Fig. 2. PAPR reduction of cyclic-SLM.

and it is related to OOB radiation [17], [18]. Actually, OOB radiation in OFDM systems consists of the inherent OFDM OOB radiation and the OOB radiation originated from the high power amplifier (HPA). The OOB comparison of the cyclicSLM for each symbol for averaging and the original OFDM signal is shown in Fig. 3 which is evaluated by using power spectral density. The OOB level with single MF [14] is $-2 \mathrm{~dB}$ more than the original signal which is shown in Fig. 3(a). It also has a ripple and spectral regrowth in the spectrum. It is seen in Fig. 3(b) that the level of the OOB with the proposed scheme is the same as the OOB of the original OFDM signal and that is around $-33 \mathrm{~dB}$. Thus, the implementation of cyclic-SLM does not cause the increase of the OOB radiation level in the OFDM signal.

\section{BER Performance}

The BER performance plots are depicted in Figs. 4(a) and 4(b). Fig. 4(a) shows the BER plot of the cyclic-SLM for perfect shifted estimation and the DC-MF scheme with $\delta=4$ and Fig.

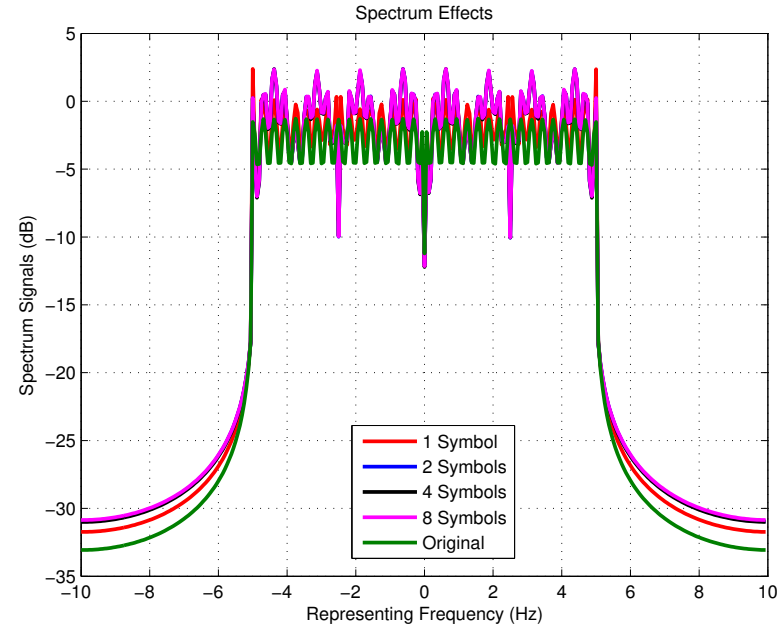

(a) OOB for single MF

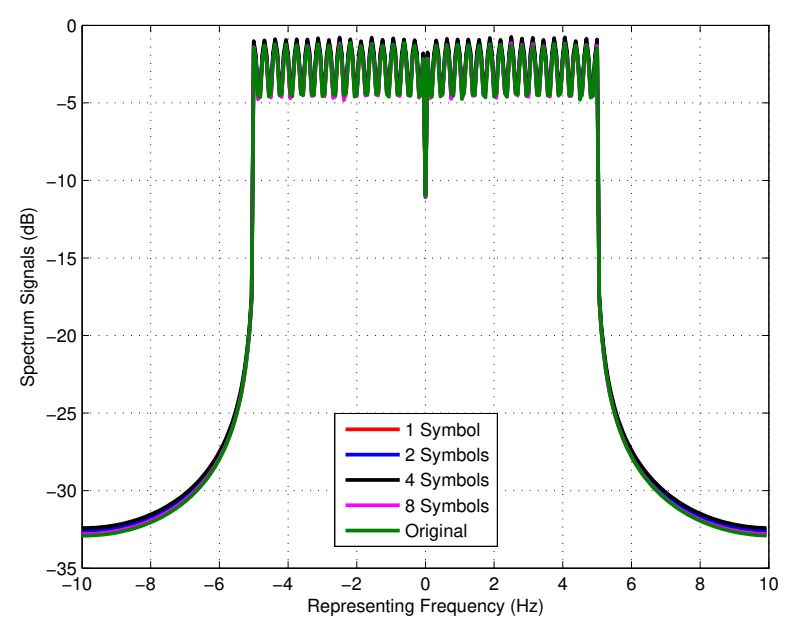

(b) OOB for multiple numbers of MF

Fig. 3. OOB Performance.

4(a) shows the same for $\delta=8$. The numbers of symbols for averaging for both plots are $1,2,4$, and 8 . It can be seen that the BER curves with $L=1,2$, and 4 show error floor. The BER difference between perfect estimation and the DC-MF scheme with the averaging of 8 symbols is about $0.8 \mathrm{~dB}$ and $0.6 \mathrm{~dB}$ in each figure.

\section{CONCLUSION}

The cyclic-SLM scheme with the DC-MF has been evaluated in this paper. We have proposed to use different phase sequence for each OFDM symbol in each frame to suppress the OOB power of the cyclic-SLM at the cost of using multiple matched filters at the receiver. Numerical results obtained through computer simulation have shown that the PAPR is significantly reduced and the use of different phase sequence and multiple matched filter reduces the OOB power to the level of the conventional OFDM. The BER performance has also been 


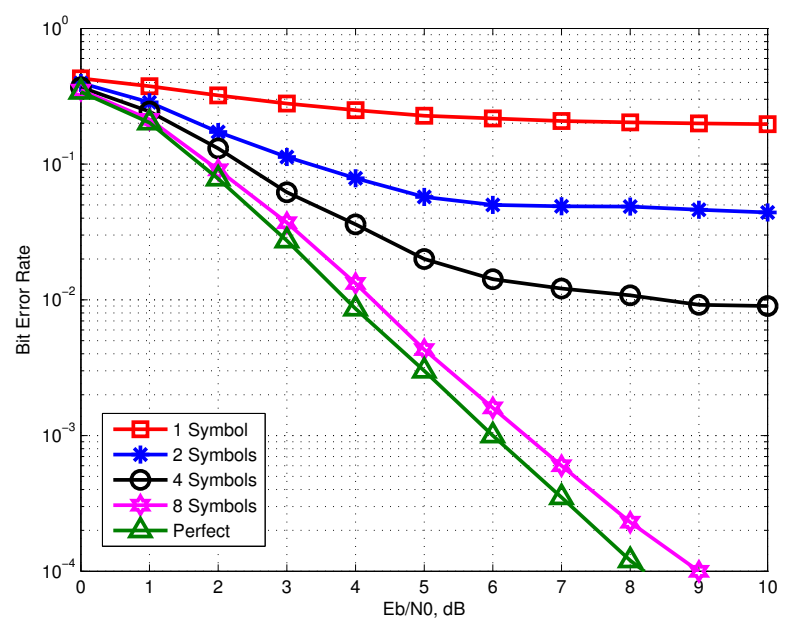

(a) Resolution of cyclic shift $\delta=4$

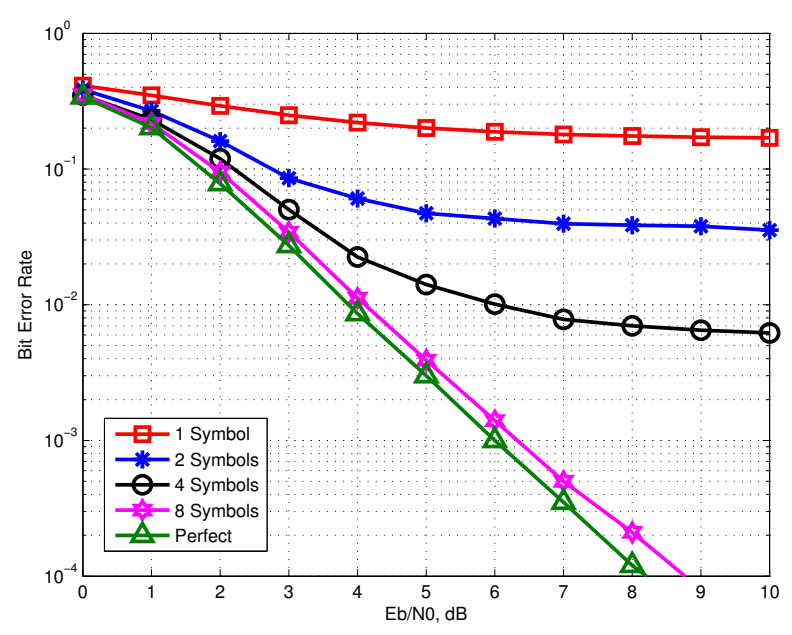

(b) Resolution of cyclic shift $\delta=8$

Fig. 4. BER on uniform delay profile channel.

evaluated. The BER plots show good performance for system with $L=8$.

\section{REFERENCES}

[1] A. M. Rateb and M. Labana, "An optimal low complexity PAPR reduction technique for next generation OFDM systems," IEEE Access, vol. 7, pp.
16406-16420, Feb. 2019.

[2] C. Balint and G. Budura, "OFDM-based multi-carrier waveforms performances in 5G," in Proc. Int. Symp. Electron. and Telecommun., Timisoara, Romania, 2018, pp. 1-4.

[3] Y. Rahmatallah and S. Mohan, "Peak-to-average power ratio reduction in OFDM systems: A survey and taxonomy," Commun. Surveys Tuts., vol. 15, no. 4, pp. 1567-1592, Fourth Quarter 2013.

[4] T. Jiang and Y. Wu, "An overview: Peak-to-average power ratio reduction technique for OFDM signals," IEEE Trans. Broadcast., vol. 54, no. 2, pp. 257-268, Jun. 2008.

[5] L. Yang, K. K. Soo, Y. M. Siu, and S. Q. Li, "A low-complexcity selected mapping scheme by use of time domain sequence superposition technique for PAPR reduction in OFDM system," IEEE Trans. Broadcast., vol. 54, no. 4, pp. 821-824, Dec. 2008.

[6] C. L. Wang and Y. Ouyang, "Low-complexity selected mapping schemes for peak-to-average power ratio reduction in OFDM systems," IEEE Trans. Signal Process., vol. 53, no. 12, pp. 4652-4660, Dec. 2005.

[7] J. S. Wang, S. H. Hwang, C. J. Kim, and Y. H. Kim, "Time-domain signal combining with cyclic delay and phase shift for PAPR reduction in OFDM system," in Proc. IEEE Int. Conf. Consum. Electron., Las Vegas, NV, USA, 2011, pp. 449-450.

[8] S. S. Eom, H. Nan, and Y. C. Ko, "Low-complexity PAPR reduction scheme without side information for OFDM systems," IEEE Trans. Signal Process., vol. 60, no. 7, pp. 3657-3669, Jul. 2012.

[9] J. Park, E. Hong, and D. S. Har, "Low complexity data decoding for SLM based OFDM systems without side information," IEEE Commun. Lett., vol. 15, no. 6, pp. 611-613, Jun. 2011.

[10] H. B. Jeon, J. S. No, and D. J. Shin, "A low-complexity SLM scheme using additive mapping sequences for PAPR reduction of OFDM signals," IEEE Trans. Broadcast., vol. 57, no. 4, pp. 866-875, Dec. 2011.

[11] S. J. Heo, H. S. Noh, J. S. No, and D. J. Shin, "A modified SLM scheme with low complexity for PAPR reduction of OFDM systems," IEEE Trans. Broadcast., vol. 53, no. 4, pp. 804-808, Dec. 2007.

[12] S. Y. L. Goff, S. S. Al-Samahi, B. K. Khoo, C. C. Tsimenidis, and B. S. Sharif, "Selected mapping without side information for PAPR reduction in OFDM," IEEE Trans. Wireless Commun., vol. 8, no. 7, pp. 3320-3325, Jul. 2009.

[13] P. D. Pamungkasari and Y. Sanada, "PAPR reduction using cyclicselective mapping with delayed correlation in time domain," in Proc IEEE Vehi. Technol. Conf. (VTC Spring), Glasgow, UK, 2015, pp. 1-5.

[14] _ _ "Time domain cyclic-selective mapping for PAPR reduction using delayed correlation with matched filter in OFDM system," in Proc. Int. Conf. Telecommun., Sydney, NSW, Australia, 2015, pp. 373-377.

[15] E. F. Badran and A. M. El-Helw, "A novel semi-blind selected mapping technique for PAPR reduction in OFDM," IEEE Signal Process. Lett., vol. 18, no. 9, pp. 493-496, Sep. 2011.

[16] P. D. Pamungkasari, Y. Sanada, F. H. Juwono, and D. Gunawan, "Cyclic shift resolution effects on OFDM system employing cyclic-SLM with delayed correlation and matched filter," in Proc. IEEE Tencon, Penang, Malaysia, 2017, pp. 153-157.

[17] K. Bae, C. Shin, and E. J. Powers, "Performance analysis of OFDM systems with selected mapping in the presence of nonlinearity," IEEE Trans. Wireless Commun., vol. 12, no. 5, pp. 2314-2322, May 2013.

[18] P. Albrecht and I. Cosovic, "On the out-of-band radiation reduction in ofdm systems," in Proc. Wireless Commun. and Netw. Conf., Las Vegas, NV, USA, 2008, pp. 559-564. 